\title{
Useful Piezoelectric Sensor to Detect False Liquor in Samples with Different Degrees of Adulteration
}

\author{
Luis Armando Carvajal Ahumada $\mathbb{D}^{1,2}$ Andrés Felipe Sandoval Cruz $\mathbb{D}^{1}{ }^{1}$ \\ Mario Alejandro García Fox, ${ }^{1}$ and Oscar Leonardo Herrera Sandoval ${ }^{1}$ \\ ${ }^{1}$ Facultad de Ingeniería y Ciencias Básicas, Universidad Central, Bogotá 110311, Colombia \\ ${ }^{2}$ Servicio Nacional de Aprendizaje (SENA), TecnoParque nodo Bogotá, Bogotá, Colombia \\ Correspondence should be addressed to Luis Armando Carvajal Ahumada; lcarvajala1@ucentral.edu.co
}

Received 12 March 2018; Revised 10 May 2018; Accepted 15 May 2018; Published 9 July 2018

Academic Editor: Alfonso Salinas-Castillo

Copyright (C) 2018 Luis Armando Carvajal Ahumada et al. This is an open access article distributed under the Creative Commons Attribution License, which permits unrestricted use, distribution, and reproduction in any medium, provided the original work is properly cited.

\begin{abstract}
The main objective of this article is to demonstrate by experimental measurements the capability of the quartz crystal resonator (QCR) to characterize samples of liquor at different concentrations of adulteration with methanol, classify them according to their viscosity, and identify how to use this capability as a potential detection of fake liquor. According to the literature, the fake liquor is a common problem detected in several countries across the globe. The current strategy to detect the fake liquor is based on a piezoelectric biosensor that permits the classification of the samples according to its viscosity. The viscosity is a useful variable to determinate the degree of adulteration in the liquor samples because the viscosity value between ethanol (straight liquor) and methanol (most common fake liquor) is very different.
\end{abstract}

\section{Introduction}

The adulterated alcohol or "fake alcohol" is a common problem detected in some countries. For instance, in the UK, the illegal alcohol is distilled and sent without a license, and its sale costs the UK around $£ 1.2$ billion per year [1]. In Greece, the improvised production of beverages named "meth" exists, done in illegal laboratories in an empirical way. The production commonly includes illegal alcohol with high concentration of methyl alcohol [2]. In India, there is a similar situation related to the illicit liquor. In these cases, one dangerous adulterant is industrial methylated spirit, which causes mass poisoning of consumers [3]. Moreover, in Czech Republic, the Czech food safety authority informed the public of persons suffering from methanol poisoning after having consumed "on tap" spirits. The product was unlabeled and supplied "in bulk" in plastic barrels [4].

In particular, the Colombian case is not an exception. Studies performed by Colombian entities such as Externado University and the National Association of Industrialists
(ANDI) reveal that the adulteration and falsification of locals have reached at $21 \%$ of the total offer. Javeriana University in another study concluded that one of each four bottles of liquors sold in Colombia has been falsified or adulterated. Moreover, Antioquia University from Colombia performed a study in which they observed that close to $50 \%$ of the liqueurs which are marketed in the Department of Antioquia, Colombia, are illegal, adulterated, or contraband [5].

In most cases, the commercial liquor is adulterated using methanol [1, 3-5], due to its low cost, and some physical characteristics are similar to those of the ethanol. Methanol $(\mathrm{CH} 3 \mathrm{OH})$ is an alcohol that is aliphatic, liquid, colorless, and volatile to room temperature. By itself, it is harmless, but its metabolites are extremely toxic. The methanol in industrial products, laboratories, and household products is commonly used. However, there is also a clandestine use like substituting of ethanol in alcoholic drinks. Although the intoxication may occur through the respiratory system or transdermally, in most cases, the exposition to methanol is through the oral way [6]. 
According to the "Report of the event notification for acute poisoning by chemical substances up to the epidemiological period XI" in 2010, out of a total of 23,844 reported intoxications, there were a total of 282 cases of methanol intoxication and 10 deaths, with a percentage of $1.18 \%$ and an incidence of 0.62 per one hundred thousand inhabitants. During 2011, 351 cases of methanol intoxication and 7 deaths were reported. Moreover, there is a high notification of consumption in the age ranging between 10 and 24 years [7]. A report of FND (National Federation of Departments) reveals that, of the percentage of illegal liquor $(24 \%)$, the $70 \%$ correspond to adulterated drinks [4].

Although some cases of intoxication by methanol are due to suicide attempts, many times, the intoxication is caused by adulterated alcohol, which generally is distributed in bottles with labels very similar to those of the original drink but with methanol mixed to the original liquor. Therefore, the adulterated drink is not detectable to naked eye [2].

The symptoms, depending on the affected system, when ingesting adulterated alcohol are [8]

(i) nausea, vomiting, abdominal pain, and later-case affections in the pancreas (gastrointestinal system),

(ii) associated manifestations of neurologic type, which in cases of lack of treatment or late detection end in a coma state (central nervous system),

(iii) decreased visual acuity until irreversible blindness due to atrophy of the optic nerve, indicating ocular involvement and representing an advanced development of the intoxication.

According to the descriptive study of methanol intoxications reported in SIVIGILA 2010-2011 in Colombia [9], when the methanol is absorbed, it undergoes oxidation processes in the organ receptor which is the liver. The enzyme responsible for this transformation is the alcohol dehydrogenase, which oxidizes it to formaldehyde, and this in turn is oxidized to formic acid (responsible for blindness) by the aldehyde dehydrogenase. The treatment generally used to treat adulterated alcohol intoxication is based on the use of ethyl alcohol to prevent the formation of toxic methanol metabolites [9].

In accordance with the problematic presentation, several techniques have been developed with the aim of detecting the adulterated liquor. The more typical laboratory techniques are the nuclear magnetic resonance spectroscopy with multivariate analysis [4] and near-infrared spectrometry (NIR) [10]. However, these techniques do not have possibilities to be used outside of the laboratory.

In Colombia, different strategies have been generated to detect the illegal liquor, divided into contraband and fake liquor cases; several years ago, for the detection of adulterated liquor, the monitoring and control program of alcoholic beverages was implemented, led by the ANDI (National Association of Industrialists) and the Ministry of Health and Social Protection with the participation of the national police, making presence in factories, warehouses, and liquor stores. For factories and warehouses, there are four visits per year, and for liquor stores, there are three visits. In these visits, the authorities make sure for sanitary conditions of the places and take random samples of a suspect batch of liquor, where these samples cannot be less than 1 liter or a bottle generally $750 \mathrm{cc}$. The first step in this process is to check the security seals and the label, the second step is to look for suspended solids inside the bottle, and the last step is to carry the sample to the corresponding authority lab and make physiochemical tests [5].

In this work, the use of a sensor based on a quartz crystal resonator in order to identify adulterated liquor in a simple and low cost-way is proposed. This sensor is capable of operating as a microviscometer knowing the density of the liquid to be analyzed [11-15]. Samples at different "levels of adulteration" have been used to get results that confirm the difference between an original alcoholic drink and adulterated one with methanol in different concentrations.

\section{Theory}

The piezoelectric sensors are considered highly efficient sensing systems not only because of their low manufacturing cost but also because of their high accuracy, biofunctionalization capacity, sensitivity, and reliability in measuring the deposited sample, either gaseous or liquid media [11].

In particular, the quartz crystal resonator (QCR) is a piezoelectric transducer sensitive to the deposition of rigid mass or liquids in contact with its surface (active electrode). The QCR is composed of two electrodes that are stimulated with AC voltage, and in consequence, the crystal vibrates. The crystal has a natural frequency of resonance that depends on its geometry and thickness; as a result, when the crystal is stimulated with a signal whose frequency is close to its natural resonance, the vibration increases. This phenomenon is visible by analyzing the behavior of the admittance or impedance of the crystal in the frequency domain $[11,16,17]$.

When the mass or liquid sample is deposited on the crystal surface, its resonance frequency changes in accordance with two equations: Sauerbrey for mass deposition (1) and Kanazawa for fluid deposition (2).

$$
\begin{gathered}
\Delta f=-\frac{2 f_{0}^{2}}{A \sqrt{\rho_{\mathrm{q}} G_{\mathrm{q}}}} \Delta m, \\
\Delta f=-\sqrt{n} f_{0}^{3 / 2} \sqrt{\frac{\rho_{\mathrm{L}} \eta_{\mathrm{L}}}{\pi \rho_{\mathrm{q}} G_{\mathrm{q}}}}
\end{gathered}
$$

where $\rho_{\mathrm{q}}$ and $G_{\mathrm{q}}$ are the specific density and the shear modulus of quartz, respectively; $f_{0}$ is the fundamental resonance frequency of the quartz, related to its thickness, and $\Delta m$ is the thin film of mass deposited; $A$ is the piezoelectrically active crystal area; $\rho_{\mathrm{L}}$ and $\eta_{\mathrm{L}}$ are the density and viscosity of the fluid, respectively; and $n$ is the overtone number [11]. 


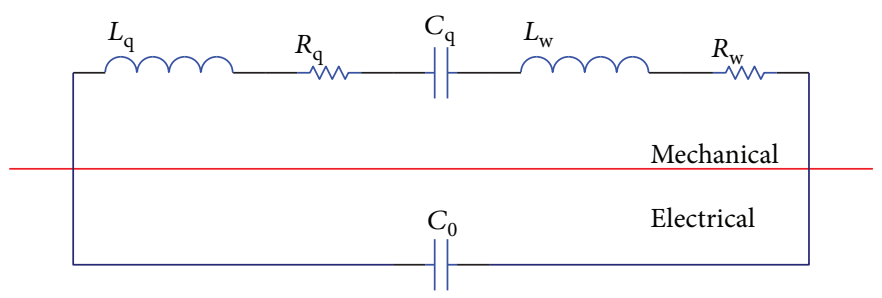

FIGURE 1: Basic electrical model (BVD) for QCM with liquid sample deposited.

In addition to Sauerbrey and Kanazawa equations, the quartz crystal behavior can be modeled as an electrical resonator circuit $[11,16,17]$.

The electric model (BVD), shown in Figure 1, explains this phenomenon as an addition of impedance depending on the type of sample. For liquids in contact with the crystal, the equivalent impedance is an inductance and a resistance which are in series.

The value of this impedance is related to the characteristics of the samples according to the following equations:

$$
\begin{aligned}
& C_{\mathrm{q}}=\frac{8 e_{26}^{2} A}{(N \pi)^{2} \overline{C_{66}} h_{\mathrm{q}}}, \\
& L_{\mathrm{q}}=\frac{1}{\omega_{\mathrm{s}}{ }^{2} C_{\mathrm{q}}}, \\
& R_{\mathrm{q}}=\frac{\eta_{\mathrm{q}}}{\overline{C_{66}} C_{\mathrm{q}}}, \\
& L_{\mathrm{w}}=\frac{\omega_{\mathrm{s}} L_{\mathrm{q}}}{N \pi}\left(\frac{2 \rho_{\mathrm{L}} \eta_{\mathrm{L}}}{\omega_{\mathrm{s}} \overline{C_{66}} \rho_{\mathrm{q}}}\right), \\
& R_{\mathrm{w}}=\frac{\omega_{\mathrm{s}} L_{\mathrm{q}}}{N \pi}\left(\frac{2 \omega_{\mathrm{s}} \rho_{\mathrm{L}} \eta_{\mathrm{L}}}{\overline{C_{66}} \rho_{\mathrm{q}}}\right), \\
& C_{0}=\frac{\varepsilon_{22} A_{e}}{h_{\mathrm{q}}},
\end{aligned}
$$

where $\overline{C_{66}}$ is the piezoelectrically stiffened elastic constant for loss-less quartz $\left(2947 \times 1010 \mathrm{~N} / \mathrm{m}^{2}\right), e_{26}$ is the piezoelectric stress constant for quartz $\left(953 \times 103 \mathrm{~A} \cdot \mathrm{s} / \mathrm{m}^{2}\right), \varepsilon_{22}$ is the quartz permittivity $\left(3.982 \times 10^{-11} \mathrm{~A}^{2} \cdot \mathrm{s}^{4} / \mathrm{kg} \cdot \mathrm{m}^{-3}\right), A$ is the active electrode area $\left(\mathrm{m}^{2}\right), h_{\mathrm{q}}$ is the quartz crystal thickness $(\mathrm{m}), \eta_{\mathrm{q}}$ is the effective viscosity of quartz $\left(3.5 \times 10^{-4} \mathrm{~kg} / \mathrm{m} \cdot \mathrm{s}\right)$, and $\omega_{s}$ is the series resonance frequency $\left(2 \pi F_{s}\right)$. For the liquid, $\rho_{\mathrm{L}}$ and $\eta_{\mathrm{L}}$ are the density of the liquid sample $\left(\mathrm{kg} / \mathrm{m}^{3}\right)$ and the viscosity of the liquid sample $(\mathrm{kg} / \mathrm{m} \cdot \mathrm{s})$, respectively [17].

Figure 2 shows the conductance behavior (Geq) for the unperturbed crystal (kept in air) and the crystal in contact with liquid. The maximum value of conductance takes place in the frequency of resonance.

According to theoretical models, when the crystal electrode is in contact with liquid samples, the conductance curve changes, moving to the left in the axis of frequency, increasing its bandwidth and decreasing its conductance magnitude (Figure 2).

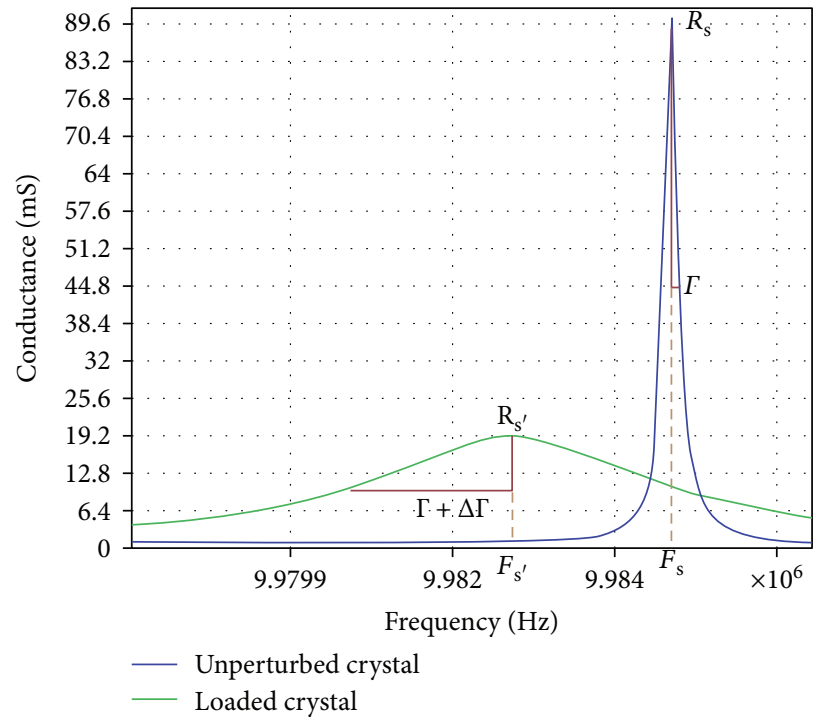

FIgUre 2: Conductance behavior for a QCM with and without the sample [12].

The values of $\Delta F$ and $\Delta \Gamma$ represent the changes in the behavior of the crystal due to the properties of the sample, and these are obtained using

$$
\begin{aligned}
\Delta F & =F_{s^{\prime}}-F_{s}, \\
\Delta \Gamma & =\Gamma^{\prime}-\Gamma, \\
\Delta R_{s} & =\frac{R_{s^{\prime}}}{R_{s}} .
\end{aligned}
$$

\section{Materials and Methods}

3.1. Samples. The experiments were done with seven adulterated samples and three references; the commercial liquor (Ron) was purchased in a local market. It has $35 \% v / v$ of alcohol concentration and was used as one of the three references for the quartz crystal resonator (QCR) system. The other two references were ethanol (99.8\% $w / w$, Merck) and methanol (99.8\% $w / w$, Merck). The commercial liquor was adulterated in the Central University laboratory, but its alcohol concentration was kept, by addition of deionized water and methanol in levels of $10 \%, 25 \%, 35 \%, 50 \%, 55 \%$, $65 \%$, and $75 \% v / v$ to the pure commercial liquor (Figure 3 ).

Only $50 \mu \mathrm{L}$ of volume of the sample was deposited in a uniform layer on the surface of the electrode of quartz crystal 


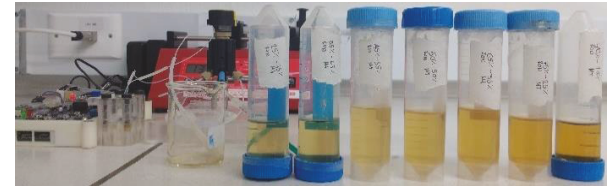

Figure 3: Samples at different concentrations elaborated in the Central University chemical lab.

to be analyzed. The deposition has been achieved using a microfluidic system.

3.2. Crystals and the Holder Cell. The $10 \mathrm{MHz}$ quartz crystals were purchased from International Crystal Manufacturing. According to the manufacturer, the crystal diameter is $13.66 \mathrm{~mm}$, the electrode diameter is $5.1 \mathrm{~mm}$, and the crystal thickness is $167 \mu \mathrm{m}$.

The holder cell has been designed and built in the mechanical laboratories at Central University in Bogotá, Colombia.

Figures $4(a)-4(c)$ show the crystal plate and the fluidic cells designed for depositing the fluid sample over the crystal electrode using a pressure pump. This technique permits the obtainment of measures related to the behavior of the crystal and the deposition of the samples with a constant flux without the need to manipulate the crystal. In this way, the reference measure is conserved. The sealing system is based on magnets, and the magnets permit a very reliable sealing and an easy way to open and close the cell.

3.3. QCR System. The development of the QCR system has been based on Nakamoto and Kobayashi's proposal [18]. In particular, the proposed circuit gets the voltage and current signal in the crystal for several frequencies in a configurable bandwidth close to the crystal resonance frequency $\left(f_{s}\right)$ indicated by the manufacturer $(10 \mathrm{MHz}$ in the current study). The current system is connected to a laptop using a software interface designed in Labview in order to control the sensor and to acquire and to visualize the signals.

All systems have been developed by the coworker team, and all technical details for the measurement system and methods to obtain the changes in the admittance curve $\left(f_{s}\right.$ and $\left.\Gamma\right)$ are described in $[11,12]$.

Figure 5(a) shows the current QCR prototype system. In future work, the prototype will be miniaturized with the aim of increasing the portability of the system, so the user can analyze the sample in any place.

Figures 5(a) and 5(b) show the microfluidic system that was used on the experiments. The system consists of two syringe pumps: the first pump injects the buffer liquid on the fluidic system and the second pump injects the sample on the fluidic system. The sample has to pass through a loop where it is stored and waits for the valve activation; when that happens, the sample can pass through the crystal.

3.4. Viscosity of the Reference. For this work, the reference viscosity values have been obtained using the viscometer Fungilab Smart (Figure 6) and the spindle LCP because the samples have low viscosity range.

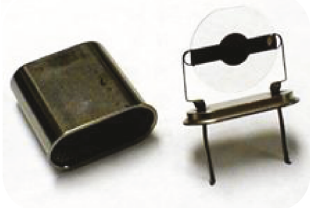

(a)

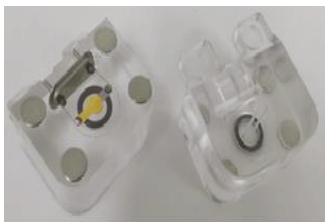

(b)

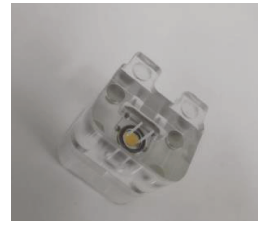

(c)
Figure 4: (a) Crystals used in experimental tests, (b) holder developed (fluidic cells in parts), and (c) holder developed (fluidic cells assembled).

3.5. Measurement Protocol. The measurement protocol is as follows: to configure the frequency sweep using the system interface (frequency range, sweep step, and number of cycles) and to calculate the series resonance frequency $\left(F_{0}\right)$ and the half-band half-width $\left(\Gamma_{0}\right)$ using the developed code in Labview for each cycle. These measures are taken ten times for the bare crystal. The final values are the averages of the ten measures. To put the sample for analysis on the electrode surface of the quartz crystal ( $50 \mu \mathrm{L}$ of volume), the microfluidic system has been used. The frequency range in the user interface needs to be adjusted in order to obtain the admittance values of the quartz crystal in contact with the liquid sample. Due to the sample effect, the curves are shifted in the frequency axis. For the results obtained in this work, the measures with the sample $\left(F_{s}, F_{r^{\prime}}, \Gamma_{s}\right.$, and $\left.R_{s^{\prime}}\right)$ are taken 100 times (ca. 3 minutes) and the final values are the averages of the measures. To obtain $\Delta F_{s}=F_{s}-F_{0}$ and $\Delta \Gamma=\Gamma_{s}-\Gamma_{0}$. The density values were obtained using an analytical balance. The mass of each solution was found from $1 \mathrm{~mL}$ of its volume.

3.6. Cleaning Protocol. Before each experiment, the sensor crystal was cleaned in $96 \%$ acetone and $99.5 \%$ isopropyl alcohol for $5 \mathrm{~min}$ each; then, it is rinsed with Milli-Q water and dried in air.

\section{Results and Discussion}

According to the measurement process described in Materials and Methods, the results obtained are the following:

Figure 7 shows the behavior of $\Delta F_{s}$ and $\Delta \Gamma$ for each sample of adulterated alcohol. According to these results, the best variable to differentiate between grades of adulteration in the alcohol sample (percentage of methanol) is $\Delta \Gamma$ due to its more sensitivity (the curve slope is higher).

Table 1 shows the results for different tests done in an independent way. One hundred measures for each test have been done. The values have been averaged. In all cases, the value $(\Delta \Gamma)$ decreases when the methanol quantity increases. In other words, the slopes for each test are similar to the 


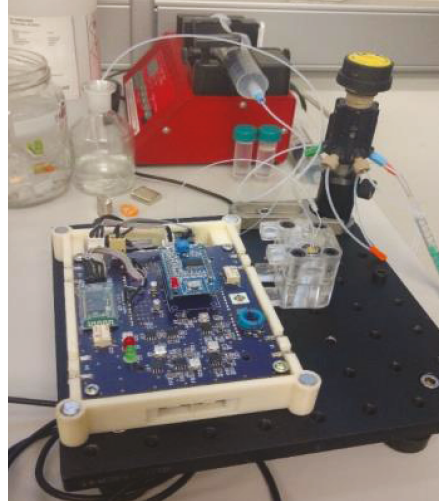

(a)

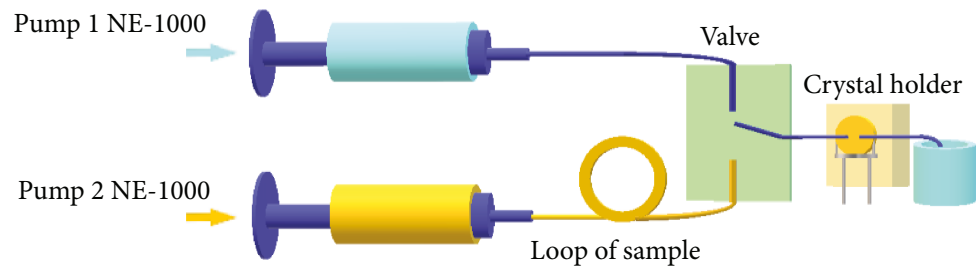

(b)

FIGURE 5: (a) QCR measurement system with the microfluidic system and holder cell and (b) schematic of the microfluidic system.

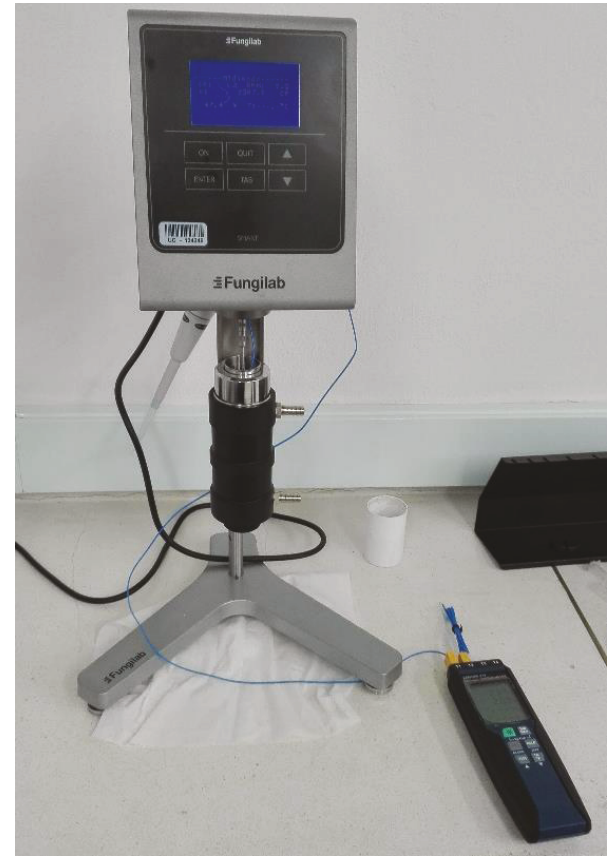

Figure 6: Viscometer for the reference (Fungilab Smart).

trend (negative slope), which indicates that the results are coherent and reproducible and they have a good level of precision. According to these results, SD is less than $10 \%$ in all cases.

Figure 8 shows the resultant curve based on the average of the tests. Error bars are calculated using the standard deviation of data tests for each mixture. According to this figure, the prototype proposed can be used to determinate the degree of adulteration of each sample in a wide range. The reason for this capability is that the piezoelectric sensors (QCR) have high sensibility for viscosity changes. In particular, the adulteration process adds methanol to the liquor mixture generating a decrease in the viscosity of the liquor in relation to a legal liquor (negative slope in Figure 9). This decrease in the viscosity value (Figure 9, gray line) is related to the resonance of the quartz crystal; in this way, reading

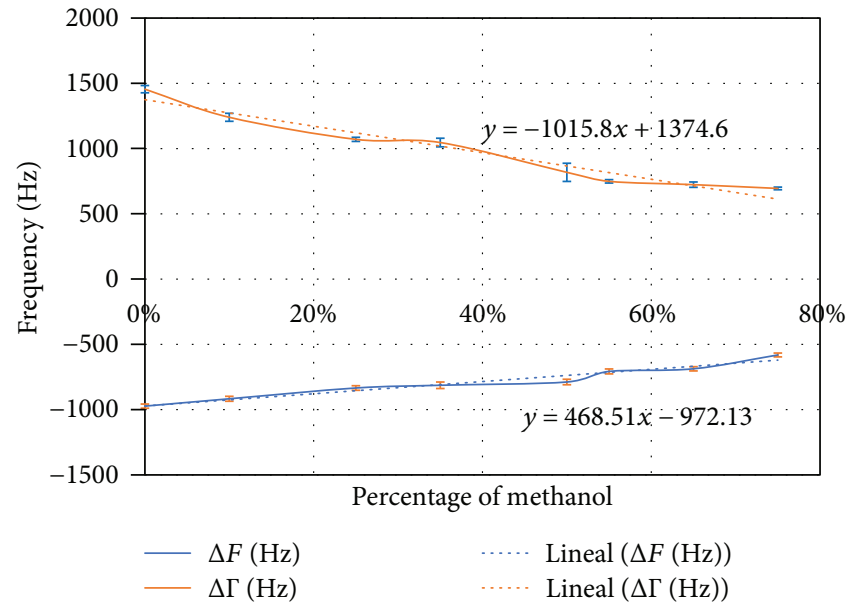

FIgURE 7: Changes in frequency for $F_{s}$ and $\Gamma$ variables for each sample.

the frequency of resonance of the quartz crystal is possible, associating the change of this frequency with the change of the viscosity of the mixture.

According to Figure 9, the values obtained for all samples using the QCR sensor have been compared with those using the commercial equipment "viscometer Fungilab Smart" available in the material laboratory at Central University. Figure 9 permits the observation of the similar behavior between the results obtained with a commercial viscometer and the values obtained in the tests using the QCR sensor. The viscosity values have been found using (2) with the data shown in Figure 9. The density values were obtained using an analytical balance. The mass of each solution was determined from $1 \mathrm{~mL}$ of its volume.

In Figure 10, the red circle zone indicates that the QCR response is better than that of the viscometer because the Fungilab measures are very close in this range $(25 \%-50 \%$ of adulterated mixture).

In accordance with the previous results, the prototype generates a very close response in comparison with the commercial equipment. In consequence, the data obtained are reliable. As an additional attribute, the quantity of the sample 
TABLE 1: Results of $\Delta \Gamma$ for four independent tests (average $\pm S D$ ). Each test contains one hundred measures.

\begin{tabular}{|c|c|c|c|c|c|c|c|c|}
\hline \multirow{2}{*}{ Methanol (\%) } & \multicolumn{2}{|c|}{ Test $1 \Delta \Gamma(\mathrm{Hz})$} & \multicolumn{2}{|c|}{ Test $2 \Delta \Gamma(\mathrm{Hz})$} & \multicolumn{2}{|c|}{ Test $3 \Delta \Gamma(\mathrm{Hz})$} & \multicolumn{2}{|c|}{ Test $4 \Delta \Gamma(\mathrm{Hz})$} \\
\hline & Average & $\mathrm{SD}$ & Average & $\mathrm{SD}$ & Average & SD & Average & $\mathrm{SD}$ \\
\hline $0 \%$ & 1374.6 & 27.87 & 1462.3 & 31.71 & 1364.6 & 29.51 & 1260.9 & 26.56 \\
\hline $10 \%$ & 1273.0 & 30.60 & 1368.7 & 33.95 & 1284.8 & 20.82 & 1187.3 & 18.06 \\
\hline $25 \%$ & 1120.7 & 15.72 & 1228.3 & 32.55 & 1165.1 & 104.91 & 1076.9 & 15.96 \\
\hline $35 \%$ & 1019.1 & 33.00 & 1134.8 & 24.98 & 1085.3 & 54.89 & 1003.3 & 34.06 \\
\hline $50 \%$ & 866.7 & 69.61 & 994.4 & 40.35 & 965.7 & 19.91 & 892.9 & 21.23 \\
\hline $55 \%$ & 815.9 & 12.94 & 947.6 & 21.22 & 925.8 & 15.86 & 856.1 & 19.26 \\
\hline $65 \%$ & 714.3 & 20.50 & 854.0 & 18.65 & 846.0 & 46.25 & 782.4 & 16.47 \\
\hline $75 \%$ & 612.8 & 10.10 & 760.4 & 14.45 & 766.2 & 21.06 & 708.8 & 41.42 \\
\hline
\end{tabular}

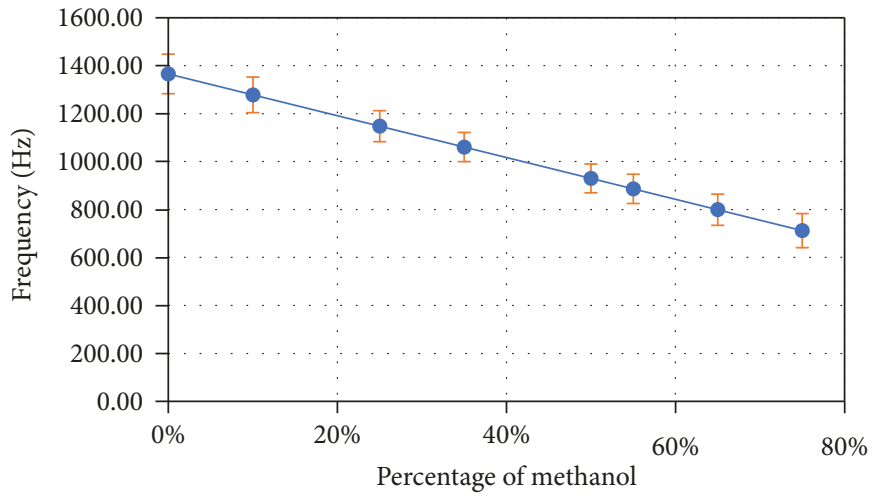

Figure 8: Average values of bandwidth shift for different independent tests.



FIGURe 9: Comparison between the sensor QCR and Fungilab Smart.

used in the comparison between the prototype QCR and the commercial equipment is tens of microliters and tens of milliliters, respectively. In consequence, the prototype proposed is more efficient about the quantities of the samples used.

\section{Conclusions}

In the present work, the effectiveness of a low-cost piezoelectric sensor to detect fake liquor has been demonstrated through simple experiments. The capability of the quartz resonator to identify changes in the viscosity of liquid samples through an electronic transduction and characterization

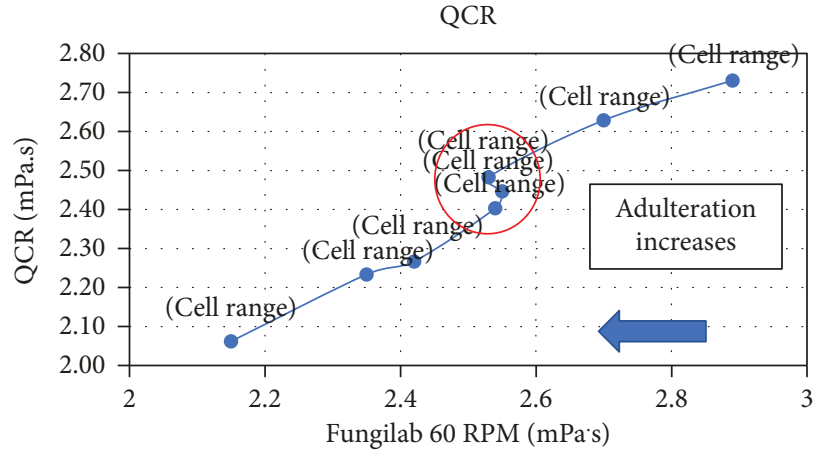

FIgURe 10: Comparison between the sensor QCR and Fungilab Smart.

of its admittance response allows us to carry out the present study to detect in a clear way the changes in concentration of methanol in a common alcoholic drink like Ron. In consequence, this device could allow the identification of adulterated alcohol with a small amount of sample (tens of microliters).

In a future phase, the device will be optimized in size, its portability will be improved, and it will be connected to a smartphone. In addition, more tests with real adulterated liquor should be conducted with the aim of verifying the presence of minimum concentrations of methanol. The real adulterated liquor samples could be obtained through an agreement with the Colombian police. 


\section{Data Availability}

Data used to support the findings of this study were supplied by Luis Carvajal under license and so cannot be made freely available. Requests for access to these data should be made to Luis Carvajal (lcarvajala1@ucentral.edu.co).

\section{Conflicts of Interest}

The authors declare that there is no conflict of interest regarding the publication of this paper.

\section{Acknowledgments}

The current investigation work has been supported by COLCIENCIAS through Grant no. 0375-2013 with project number 6570577636375 and by Universidad Central (Bogotá, Colombia) through the Research Cluster on Converging Sciences and Technologies (NBIC).

\section{References}

[1] T. Robert, Combatting Fake, Counterfeit, and Contraband Alcohol Challenges in the United Kingdom through the Alcohol Wholesaler Registration Scheme (AWRS), Federal Regulatory and Compliance Consultant Falls Church, Virginia, 2017.

[2] A. Tsiboukli, "Greek crisis: impact on drug treatment services, research and population," Nordic Studies on Alcohol and Drugs, vol. 32, no. 3, pp. 333-337, 2015.

[3] L. Riley, M. Marshall, World Health Organization, and Dept. of Substance Abuse, Alcohol and Public Health in 8 Developing Countries, World Health Organization, Geneva, Switzerland, 1999.

[4] D. W. Lachenmeier, Advances in the Detection of the Adulteration of Alcoholic Beverages Including Unrecorded Alcohol, Elsevier Ltd, 2016.

[5] J. Zapata, A. Sabogal, A. C. Montes, G. Rodriguez, and J. Castillo, Una Estimación de la Adulteración y la Falsificación de Bebidas Alcohólicas en Colombia, Fedesarrollo, 2012.

[6] J. L. Castellanos, A. B. Limón, A. R. Hernández, and D. E. F. Rojas, "Intoxicación por metanol, reporte de un Caso," Archivos de Medicina de Urgencia de México, vol. 1, pp. 67-73, 2009.

[7] Departamento Nacional de Planeación - DNP, "Estrategia para la Implementación del Sistema Unico Nacional de Información y Rastreo -SUNIR (Documento Conpes)," Consejo Nacional de Política Económica y Social República de Colombia, p. 33, 2009.

[8] Methanol Institute, "Methanol FACTS: adulterated alcohol poisoning: issue summary," 2016, http://www.methanol. org/wp-content/uploads/2016/06/Adulterated-Alcohol-IssueSummary-20160322-EN-vfinal.pdf.

[9] D. Niño and J. Velandia, Estudio Descriptivo De Las Intoxicaciones Por Metanol Reportadas En Sivigila 2010-2011 En Colombia, Universidad de Ciencias Aplicadas y Ambientales (UDCA), 2014.

[10] M. J. C. Pontes, S. R. B. Santos, M. C. U. Araújo et al., "Classification of distilled alcoholic beverages and verification of adulteration by near infrared spectrometry," Food Research International, vol. 39, no. 2, pp. 182-189, 2006.

[11] L. A. Carvajal Ahumada, N. Peña Pérez, O. L. Herrera Sandoval, F. del Pozo Guerrero, and J. J. Serrano Olmedo, "A new way to find dielectric properties of liquid sample using the quartz crystal resonator (QCR)," Sensors and Actuators A: Physical, vol. 239, pp. 153-160, 2016.

[12] L. Ahumada, M. González, O. Sandoval, and J. Olmedo, "Evaluation of hyaluronic acid dilutions at different concentrations using a quartz crystal resonator (QCR) for the potential diagnosis of arthritic diseases," Sensors, vol. 16, no. 11, p. 1959, 2016.

[13] L. A. Carvajal Ahumada, J. E. Pazos Alonso, J. J. Serrano Olmedo, and O. L. Herrera Sandoval, "Diseño y Evaluación de un micro viscosímetro de bajo costo utilizando un resonador de cristal de cuarzo y Arduino," in Congreso Internacional de Ciencias Básicas e Ingeniería de Universidad de los Llanos, p. 11, Asociación Mexicana de Medicina de Urgencia, 2016.

[14] E. J. Calvo, R. Etchenique, P. N. Bartlett, K. Singhal, and C. Santamaria, "Quartz crystal impedance studies at 10 $\mathrm{MHz}$ of viscoelastic liquids and films," Faraday Discussions, vol. 107, pp. 141-157, 1997.

[15] M. Pax, J. Rieger, R. H. Eibl, C. Thielemann, and D. Johannsmann, "Measurements of fast fluctuations of viscoelastic properties with the quartz crystal microbalance," Analyst, vol. 130, no. 11, pp. 1474-1477, 2005.

[16] M. Cassiède, J. H. Paillol, J. Pauly, and J.-L. Daridon, “Electrical behaviour of AT-cut quartz crystal resonators as a function of overtone number," Sensors and Actuators A: Physical, vol. 159, no. 2, pp. 174-183, 2010.

[17] V. E. Granstaff and S. J. Martin, "Characterization of a thickness-shear mode quartz resonator with multiple nonpiezoelectric layers," Journal of Applied Physics, vol. 75, no. 3, pp. 1319-1329, 1994.

[18] T. Nakamoto and T. Kobayashi, "Development of circuit for measuring both $\mathrm{Q}$ variation and resonant frequency shift of quartz crystal microbalance," IEEE Transactions on Ultrasonics, Ferroelectrics and Frequency Control, vol. 41, no. 6, pp. 806-811, 1994. 


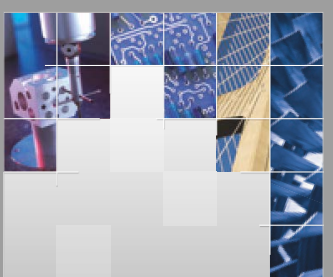

\section{Enfincering}
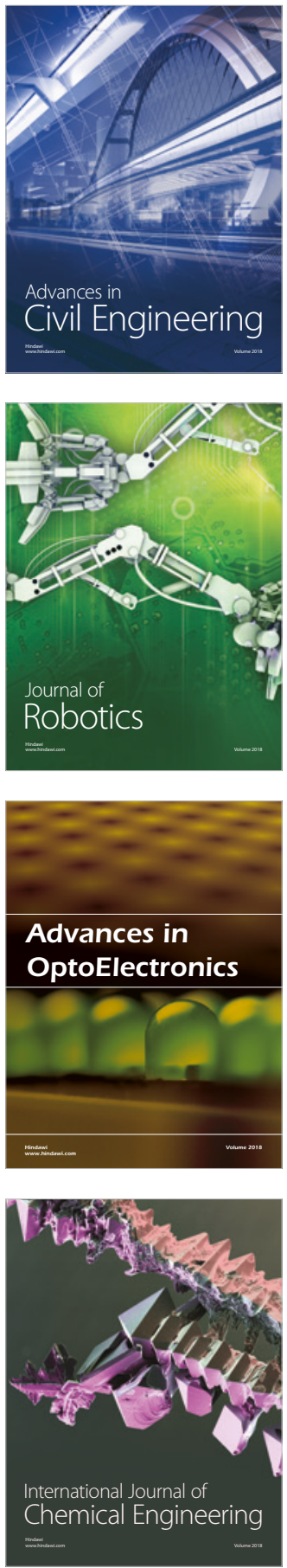

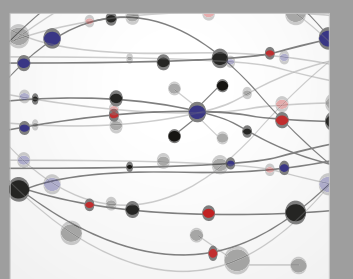

\section{Rotating \\ Machinery}

The Scientific World Journal

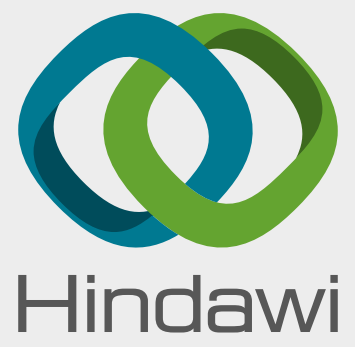

Submit your manuscripts at

www.hindawi.com
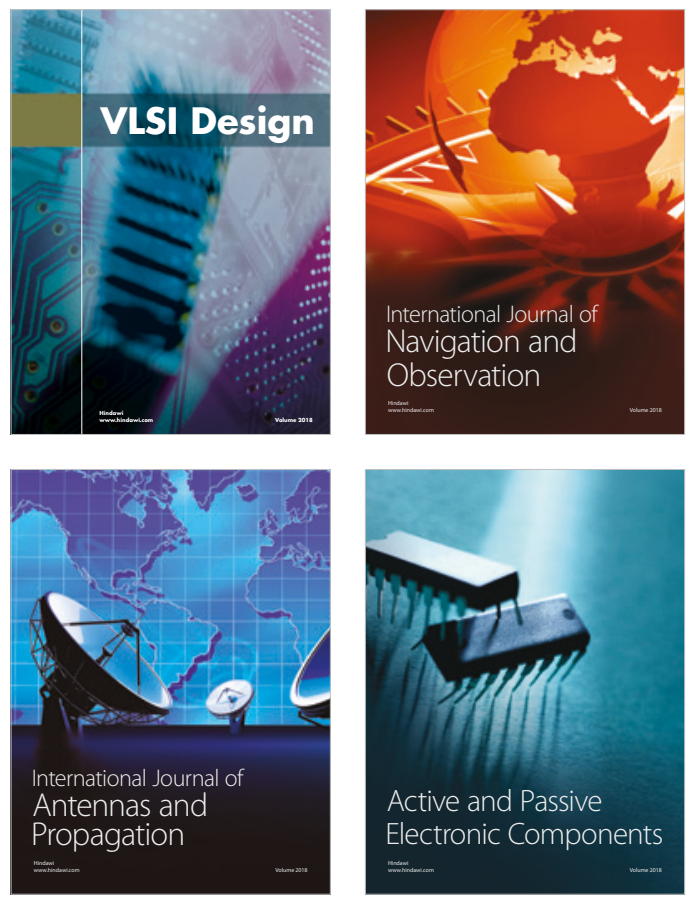
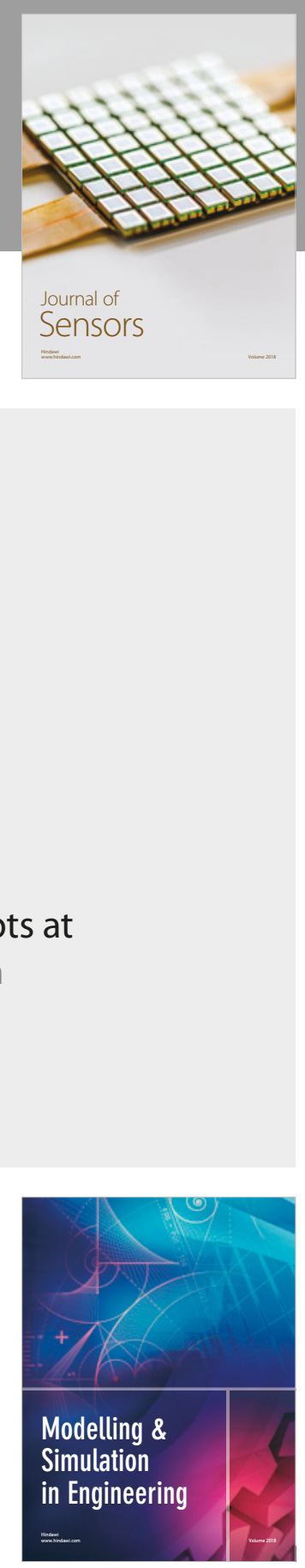

\section{Advances \\ Multimedia}
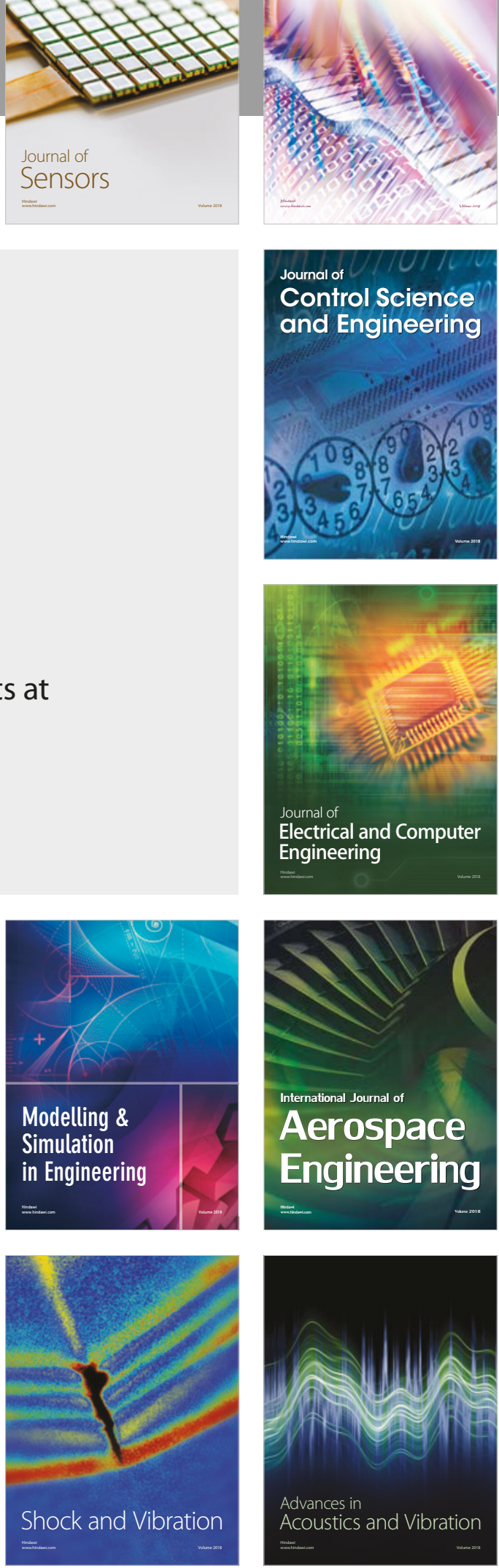